\title{
Application Article \\ New Flexible Medical Compact Antenna: Design and Analysis
}

\author{
Yann Mahe, Anne Chousseaud, Marc Brunet, and Bruno Froppier \\ Lunam Université de Nantes, UMR 6164 Institut d'Electronique et de Télécommunications de Rennes, Site de Nantes, \\ Polytech Nantes, rue Christian PAUC, BP 50609, 44306 Nantes Cedex 3, France \\ Correspondence should be addressed to Yann Mahe, yann.mahe@univ-nantes.fr
}

Received 14 December 2011; Revised 16 February 2012; Accepted 19 February 2012

Academic Editor: Hendrik Rogier

Copyright (C) 2012 Yann Mahe et al. This is an open access article distributed under the Creative Commons Attribution License, which permits unrestricted use, distribution, and reproduction in any medium, provided the original work is properly cited.

\begin{abstract}
Some results on embedded antennas for medical wireless communication systems are presented. Medical telemetry can advantageously assist medical diagnostics. For example, you can better locate a diseased area by monitoring temperature inside the human body. In order to establish efficient wireless links in such an environment, a special attention should be paid to the antenna design. It is required to be of a low profile, very small regardless of the working frequency- $434 \mathrm{MHz}$ in the ISM band, safe, and cost effective. Design of the as-considered antenna is proposed based on a simple model. The approach has been demonstrated for a compact flexible antenna with a factor of 10 with respect to the half-wave antenna, rolling up inside an ingestible pill. Measured and calculated impedance behaviour and radiation characteristics of the modified patch are determined. Excellent agreement was found between experiment and theory.
\end{abstract}

\section{Introduction}

Medical applications benefited from recent research in the field of miniaturized wireless communication systems. Depending on the working frequencies, one of the main challenges is to design very small antennas; that would be to make these communication systems implantable or ingestible in the human body. Various types of compact antennas have been investigated [1-7]. Nevertheless, it is sometimes quite difficult to design the same kind of proposed antennas when the working frequency or the available space is different. Indeed, no design rules have been given to help the engineers.

In the case of digestive monitoring system, an ingestible capsule with a small size is required. Therefore, all monitoring systems must be implemented inside the desired pill.

For example, we can retain a classical used pill as shown in Figure 1. The space allowed is very limited. This is especially true when not only the antenna but also other active parts (batteries, sensors, etc.) of the communication system are embedded within a pill.

Moreover, these parts have to be shielded from a radiation that can interfere with the sensor and generate erroneous data.

The dimensions of the selected pill are $7 \mathrm{~mm}$ diameter and $17 \mathrm{~mm}$ height. So, we propose to investigate a microstrip patch antenna for which a particular effort in terms of miniaturization must be done. Two main points support this decision. Firstly, this kind of antenna presents a ground plane on its bottom face which can advantageously be used for shielding the electronic part of the module from radiation. Secondly, if this antenna is manufactured on a flexible substrate, it can easily be rolled up inside the pill.

The retained frequency for this specific application is from the ISM bandwidth $(433.05 ; 434.79 \mathrm{MHz})$ in which equivalent isotropic radiated power (EIRP) must be lower than $14 \mathrm{dBm}$. So the main challenge is to reduce the size of the antenna for about a factor of $90 \%$ compared with classical half-wavelength patch antenna and to ensure the conformability inside the pill.

Herewith, we will start by presenting the antenna geometry and the design rules to obtain an initial structure close to the optimal one. Our aim is to provide a very simple model in order to limit the optimization effort.

This approach proves its efficiency because the length of the proposed antenna for first estimation and final solution is slightly modified.

A first didactic example of low-cost antenna in free space on FR4 dielectric $\left(\varepsilon_{r}=4.4\right.$ and height $\left.=1.58 \mathrm{~mm}\right)$ is proposed. 


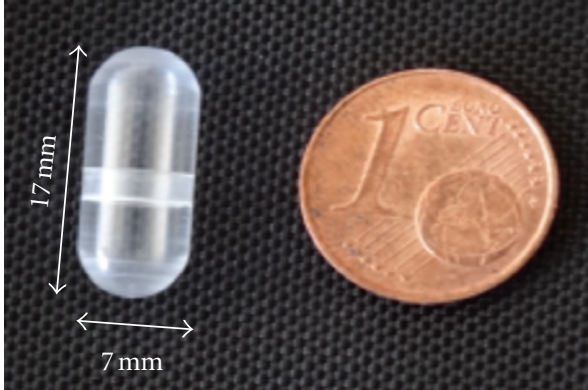

Figure 1: Example of possible pill.

This structure has been optimised with CAD simulation tool like Ansoft HFSS. Scattering parameter $\left(S_{11}\right)$ shows a good agreement between simulation and measurement. The input impedance of the antenna can take any value between 0 and a maximum value fixed by the geometry. To illustrate this concept, an example of $25 \Omega$ input impedance antenna is proposed. This input impedance can be easily changed by choosing an appropriate position of the feeding point along the structure. Finally, the dimensions of a medical folding antenna is proposed and investigated. All are within an environment whose properties are close to those of the human body with concluding results.

The preliminary design method will be described in detail below.

\section{Theoretical Considerations}

2.1. Physical Analysis of Field Distributions. Patch antennas (Figure 2) are very common structures, but their size is one of the main drawbacks (half guided wavelength size). As long as the patch antenna works on its $\mathrm{TM}_{01}$ mode, it is possible to state that the electrical field has constant amplitude along its width $(W)$ and varies in cosine along its length $(L)$. A common technique to reduce the overall size of a microstrip patch antenna is to use only half part of this antenna placing some shorting points on the null electric field plane. The field distribution remains unchanged in both the cases. In this configuration (Figure 2(b)), the antenna length corresponds approximately to a quarter guided wavelength [8].

Introducing one or more impedance steps in the structure (Stepped Impedance Resonator, SIR Concept, Figure 3 ) will lead to either one of the following: a nonperiodic frequency behaviour with nonharmonic values resonant frequencies $[9,10]$ or smaller structures without changing their fundamental working frequency. One typical example for this is the H-antenna $[11,12]$ obtained from a half-wave structure. An example to reduce the overall size of such antenna would be to combine the two previous techniques as shown in Figure 4.

Finally, if the smallest line width is long enough in this last structure, it is possible to further reduce the length of the antenna by meandering it. The concept of using these three miniaturization techniques is very appealing in terms of compactness. However, it would be more interesting if one can determine approximate dimension of such a structure in a simple way.

2.2. Design Method. It is well known that resonance conditions are achieved when the imaginary part of the equivalent input impedance of a resonator is null. In case of short-ended SIR antenna, by neglecting the discontinuity effects, it is possible to propose a simple model for this kind of structure. The SIR antenna is now reduced to two transmission lines with different characteristic impedances, ended with one part by a short circuit and the other part by an open circuit. This model is presented in Figure 5.

The impedance $Z_{\text {input }}$ at a distance $L$ from a given impedance load through a lossless transmission line with an homogeneous characteristic impedance $Z_{c}$ and a wave number $\beta$ is given below

$$
Z_{\text {input }}=Z_{c} \cdot \frac{Z_{\text {load }}+j \cdot Z_{c} \cdot \tan (\beta \cdot L)}{Z_{c}+j \cdot Z_{\text {load }} \cdot \tan (\beta \cdot L)} .
$$

This equation can be applied to both sections of the proposed design. So, for the structure described in Figures 3 and 4, the equivalent impedance $Z_{r 1}$ presented at the end of the first line section $\left(W_{1}, L_{1}\right)$ loaded by an open circuit is given as follows:

$$
Z_{r 1}=\frac{-j \cdot Z_{c 1}}{\tan \left(\beta_{1} \cdot L_{1}\right)} .
$$

Through the second line ( $\left.W_{2}, L_{2}\right)$, the equivalent impedance can be calculated by substituting $Z_{r 2}$ equal to zero on the short cut plane (short circuit) at the working frequency:

$$
Z_{r 2}=Z_{c 2} \cdot \frac{Z_{r 1}+j \cdot Z_{c 2} \cdot \tan \left(\beta_{2} \cdot L_{2}\right)}{Z_{c 2}+j \cdot Z_{r 1} \cdot \tan \left(\beta_{2} \cdot L_{2}\right)}=0 .
$$

We can get the condition of resonance (4) by solving (2) and (3) which will lead us to a two-equation system given by (5).

$$
\begin{gathered}
Z_{c 2} \cdot \frac{-j \cdot Z_{c 1}+j \cdot Z_{c 2} \cdot \tan \left(\beta_{2} \cdot L_{2}\right) \cdot \tan \left(\beta_{1} \cdot L_{1}\right)}{Z_{c 2} \cdot \tan \left(\beta_{1} \cdot L_{1}\right)+Z_{c 1} \cdot \tan \left(\beta_{2} \cdot L_{2}\right)}=0, \\
-Z_{c 1}+Z_{c 2} \cdot \tan \left(\beta_{2} \cdot L_{2}\right) \cdot \tan \left(\beta_{1} \cdot L_{1}\right)=0, \\
Z_{c 2} \cdot \tan \left(\beta_{1} \cdot L_{1}\right)+Z_{c 1} \cdot \tan \left(\beta_{2} \cdot L_{2}\right) \neq 0 .
\end{gathered}
$$

Here, we have to find the optimal value of the quadruplet $\left(Z_{c 1}, Z_{c 2}, L_{1}, L_{2}\right)$ which satisfies the conditions given by (5). Equation (5) represents an overdefined system. This leads designers to choose which variable should be fixed first.

Since the problem is related to compactness, the width and the length of the first part have to be first chosen.

An important effort of miniaturization is made by meandering the high impedance line. To improve this effect, the second line width has to be compatible with meandering, and it also should be fixed.

By setting the values of $W_{1}, W_{2}$, and $L_{1}$, it is possible to determine the length $L_{2}$ for which the given operating frequency is achieved. For example, if we choose $W_{1}=18 \mathrm{~mm}$, 


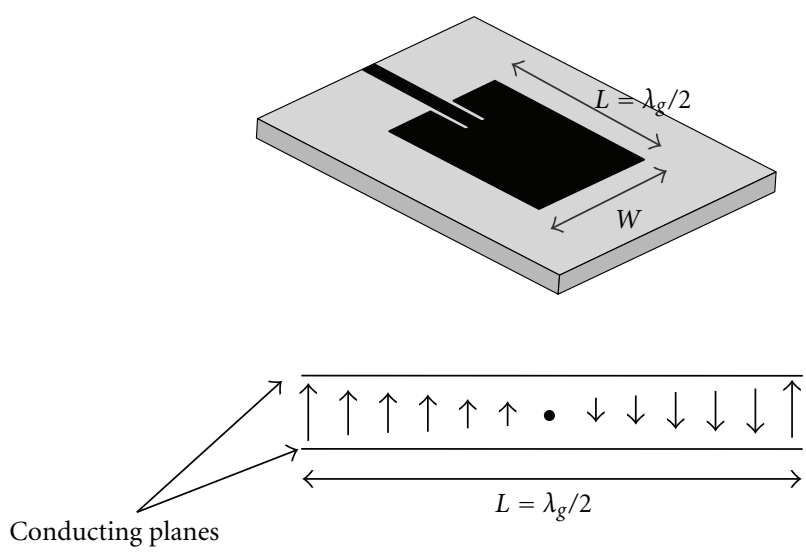

(a)
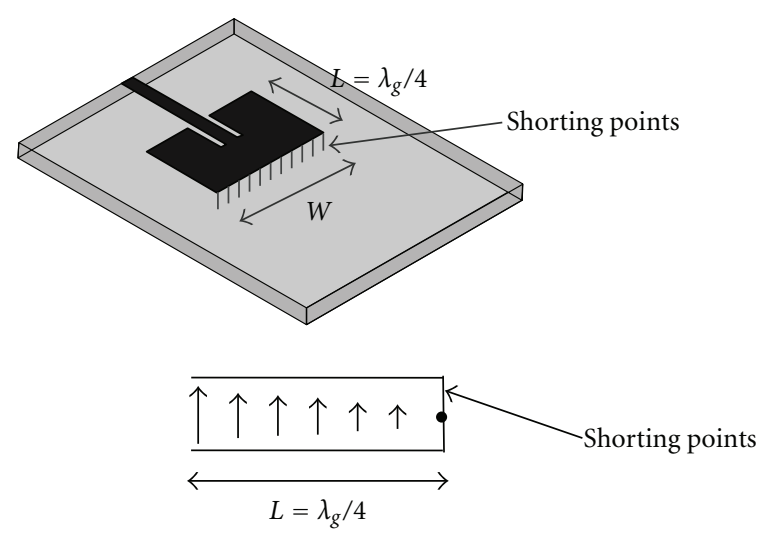

(b)

FIGURE 2: Example of elementary half (a) and quarter wave (with shorting points) (b) patch antennas. Electric field distributions along the antenna lengths.

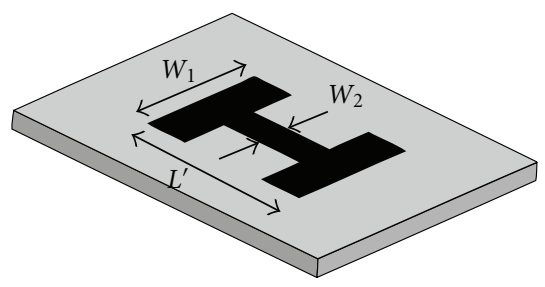

FIgURe 3: $\mathrm{H}$ antenna example.

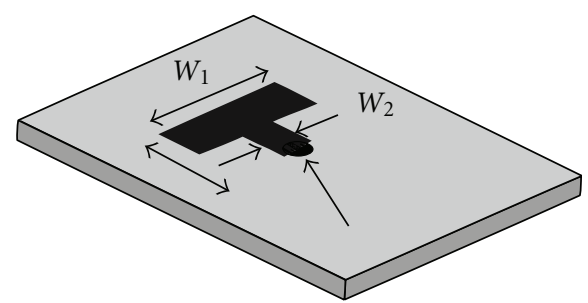

FIgURE 4: Short-circuited SIR antenna.

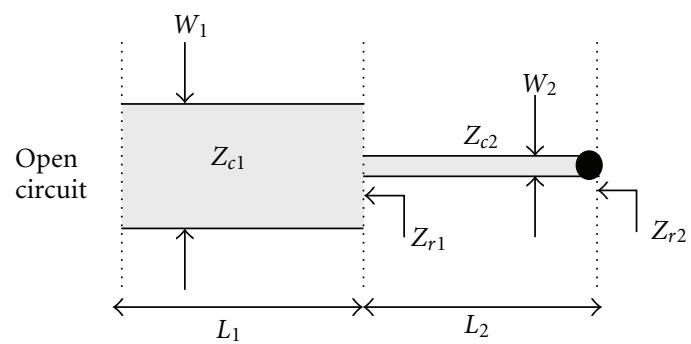

Figure 5: Description of the structure.

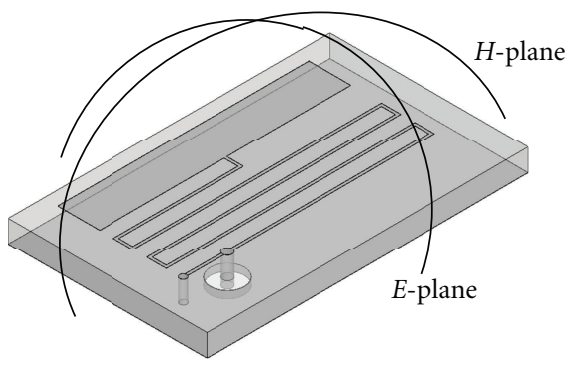

Figure 6: Final antenna.
$L_{1}=3 \mathrm{~mm}$, and $W_{2}=0.2 \mathrm{~mm}$, then $L_{2}$ can be calculated as $67 \mathrm{~mm}$ for the resonant frequency of $434 \mathrm{MHz}$.

As the difference in width goes higher, more importance will be placed on compactness.

To further increase the compactness, the second line section can be meandered according to the width of the first section line (Figure 6). The gap between meandered lines has to be adjusted to have a capacitive coupling between arms as low as possible. Thus, the chosen gap will be five times greater than its width. The feeding point along the antenna is dependent on the input impedance chosen for the antenna. Indeed, the antenna can be matched directly on any real impedance under a maximum value determined by the geometry. An HFSS simulation is also required using $L_{2}$ as the only optimization parameter.

\section{Results}

3.1. First Realization on Rigid and Low-Cost Substrate. According to the design rules previously presented, a free space antenna built on FR4 substrate is fabricated. A gap between two meandered lines is chosen equal to $1 \mathrm{~mm}$ as mentioned before. This structure is simulated and optimized using Ansoft HFSS. Optimal dimensions are $18 \mathrm{~mm} \times 9 \mathrm{~mm}$ with the first line section dimension of $3 \times 18 \mathrm{~mm}^{2}$ and for the second one $0.2 \times 75 \mathrm{~mm}^{2}$. The $L_{2}$ length is shifted $8 \mathrm{~mm}$ from formula compared with simulations and measurements results. This could be explained by discontinuities effects along the antenna which are not taken into account in the simple used model. 


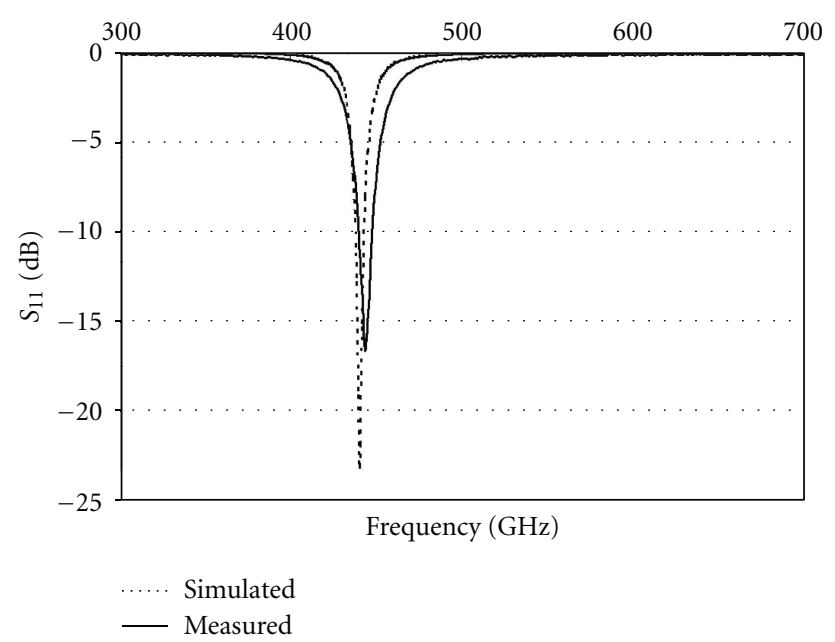

FIGURE 7: $S_{11}$ coefficient (dotted lines: HFSS simulation, solid lines: measurement) for $25 \Omega$ access.

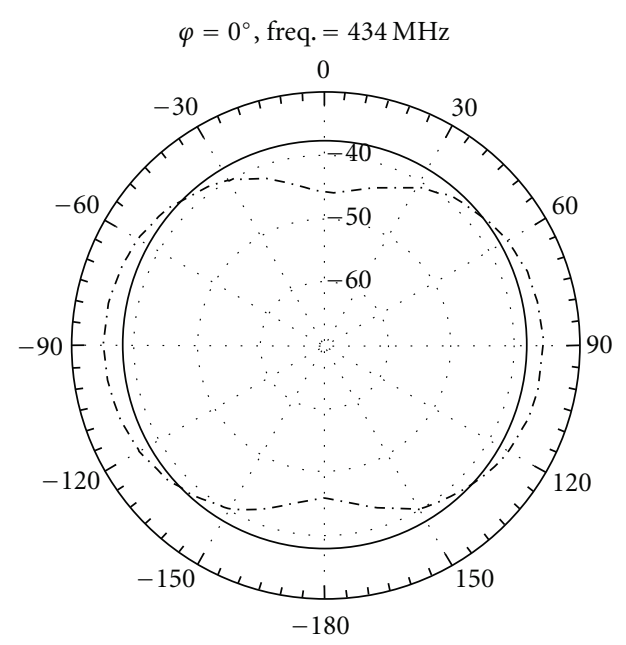

(a)

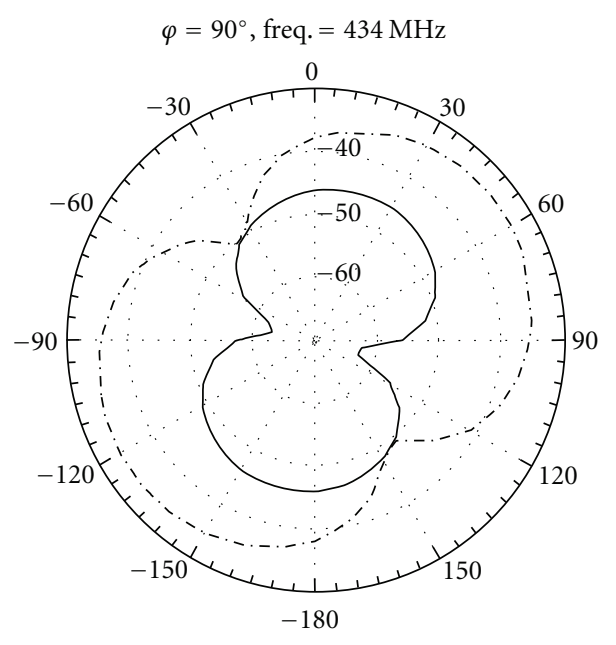

(b)

Figure 8: Radiation pattern $G_{\varphi}$ (dot dash) and $G_{\theta}$ (solid) at $434 \mathrm{MHz}$ and $25 \Omega$ access as simulated.
The design of the proposed antenna is given in Figure 6. The input impedance chosen for the antenna is, for example, $25 \Omega$, but it can vary from 0 up to $6 \mathrm{k} \Omega$ in this topology.

Measurements of scattering parameter $S_{11}$ are in a good agreement with simulations as shown in Figure 7.

Radiation pattern is given in Figure 8 in two characteristic planes. Maximum radiation gain is around $-33 \mathrm{dBi}$, with a radiation pattern quite omnidirectional in the $\varphi=0$ plane. The gain can be improved by increasing the length of the $L_{1}$ section but at the expense of compactness.

We have proposed an antenna design method with strong constraint in terms of compactness. This method is based on the prior knowledge of characteristic impedance and propagation constant values of the two line sections. This information could be evaluated by the formula given by [13]. The length of $L_{2}$ is then evaluated using (5) to fix the resonant frequency. This method has been applied to design a conformal antenna for medical application in an environment whose properties are close to those of the human body. In this type of application, a flexible substrate is suitable to roll in the antenna inside a pill.

3.2. Realization on Flexible Substrate. To ensure compactness constraints of ingestible pills, the design has been carried out on a $50 \mu \mathrm{m}$ thickness flexible substrate (AP8525 from DuPont Pyralux) with a metal thickness of $18 \mu \mathrm{m}$ and a relative permittivity of 3.4.

Antenna dimensions are evaluated based on the previous design rules.

As stated before, the method is based on the prior knowledge of characteristic impedance and propagation constant values for each line sections. These values are thus computed by HFSS modelling each line cross-section. Fixing the width and the length of the first line section $\left(18 \times 3 \mathrm{~mm}^{2}\right)$ and the width of the second one $(0.2 \mathrm{~mm})$, the length $L_{2}$ could be calculated according to (5). The computed length $L_{2}$ is found equal to $22.5 \mathrm{~mm}$. As in the case of the first studied antenna, a shift of $6.5 \mathrm{~mm}$ from formula compared with simulation and measurement results is observed. The second line section dimensions are therefore $0.2 \times 16 \mathrm{~mm}^{2}$. Dimensions of the antenna built on flex are $18 \times 5.4 \mathrm{~mm}^{2}$ which is completely compatible with the dimensions of the selected pill.

A prototype of the antenna has been fabricated and investigated according to the measurement setup described in Figure 9. This consists of the antenna under test (1) immersed in a $20 \mathrm{~cm}$ diameter container (2) full of specific human tissue equivalent liquid material with a dielectric constant of 49.6 and a conductivity of $0.51 \mathrm{~S} / \mathrm{m}$. The receiver (3), $80 \mathrm{~cm}$ aside from the container, consists of a halfwave vertical polarized patch antenna ( $5 \mathrm{dBi}$ gain) associated with low noise amplifier (LNA) to be as close as possible to the target application. The reflection and transmission coefficients were measured by a vector network analyzer (VNA) (4) operating over the range of $300 \mathrm{kHz}-20 \mathrm{GHz}$ with $50 \Omega$ RF input impedance.

Measurement results show a good matching with simulation in terms of frequency setting and scattering parameters (Figure 10). The link budget (transmission coefficient) has 


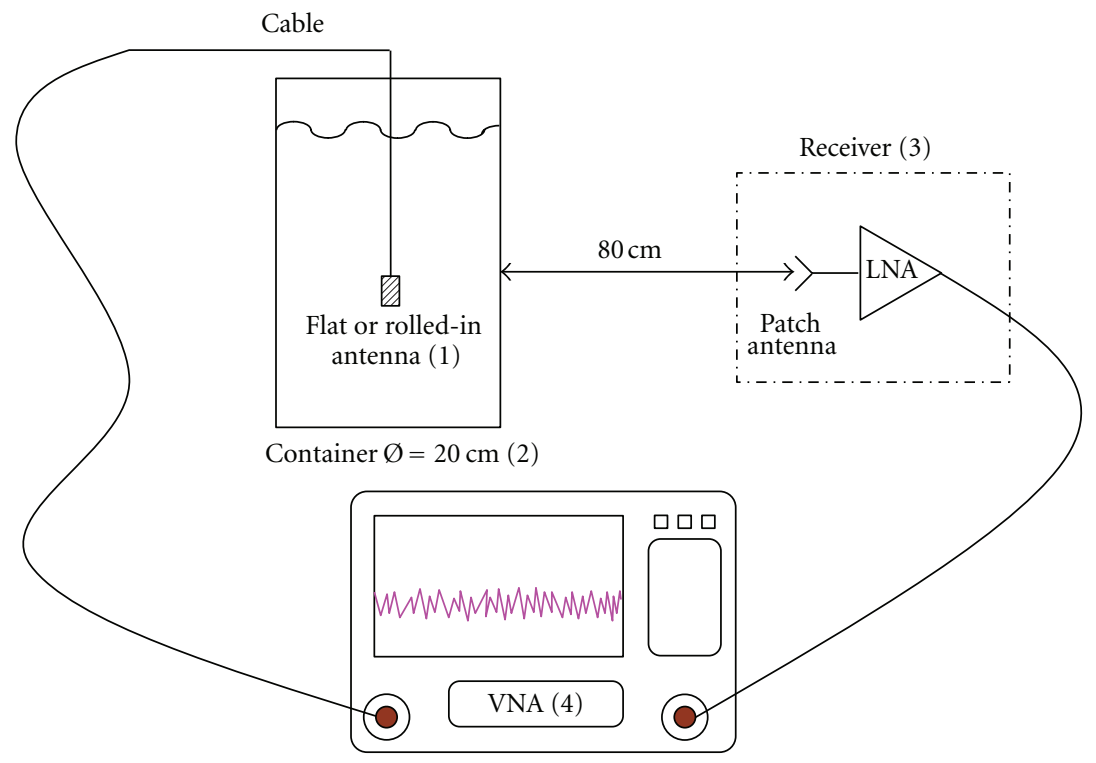

FIGURE 9: Structure of the system.

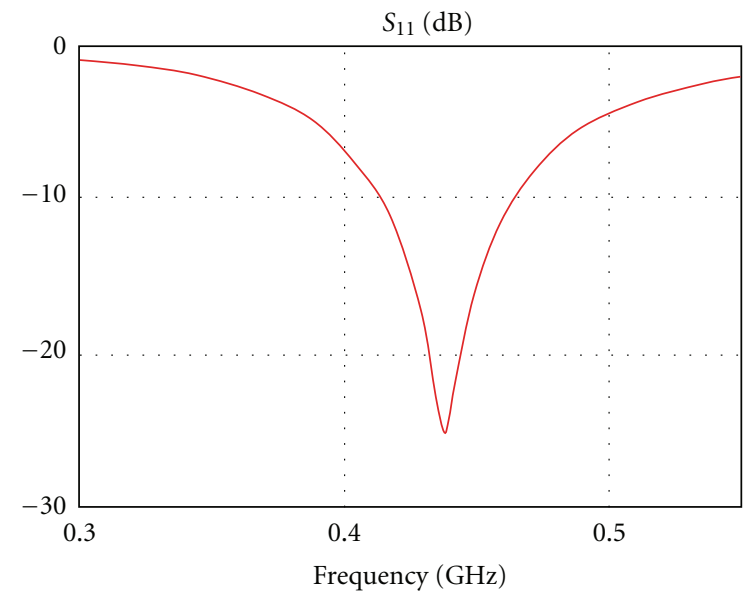

(a)

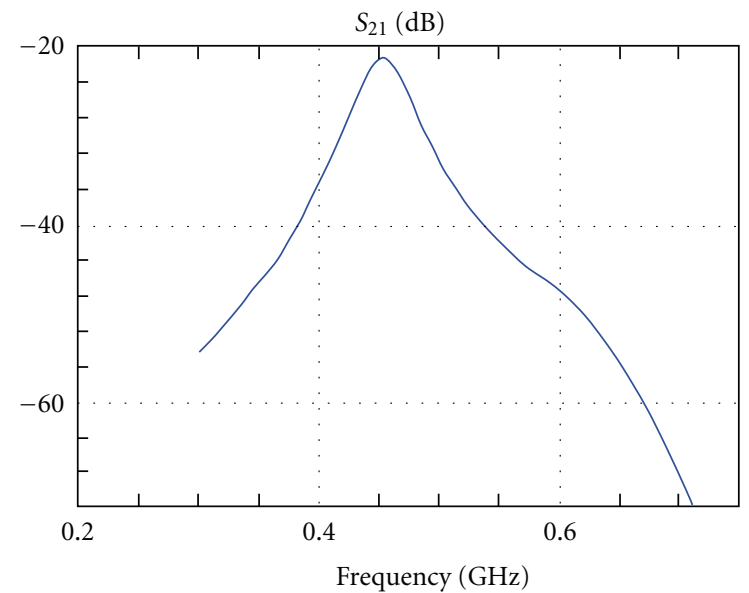

(b)

Figure 10: Measured scattering parameter of the antenna deep in tissue-equivalent liquid material. been measured when the antenna is rolled in or not in the pill. No major change has been observed between the two configurations. Moreover, the radiation of the proposed antenna is quite omnidirectional. Different configurations have been tested, and the budget link is slightly modified (less than $3 \mathrm{~dB}$ over $360^{\circ}$ range).

To improve the link budget, one can actually imagine a receiver placed on the human body interface. In this case, the received antenna should be designed properly taking into consideration the human body impact.

A first prototype of the final system is presented in Figure 11. The antenna is rolling up inside the capsule, and therefore the active part is shielded by the ground plane of the antenna.

\section{Conclusion}

In this paper, we have demonstrated that a very compact antenna $\left(\lambda_{\mathrm{g}} / 20\right)$ can be obtained using several miniaturization techniques. The dimensions of the design have been calculated from a simple theoretical model. The difference between first estimation and final solution is minimal which proves the approach efficiency. To illustrate this concept, we have designed an antenna working around $434 \mathrm{MHz}$ frequency. An antenna of size $18 \mathrm{~mm} \times 9 \mathrm{~mm}$ is designed compared with classical half guided wavelength patch antenna ( $20 \mathrm{~cm}$ on FR4 substrate). A size reduction of the overall antenna of about $90 \%$ is achieved. Any arbitrary real input impedance from 0 up to few $\mathrm{k} \Omega$ can be chosen by finding appropriate position of the feeding point along the structure. Simulations and measurements match well whereas radiation pattern is quite omnidirectional in one plane. This antenna has also been designed on a flex substrate to be in terms embedded as a conformal antenna in different applications like ingestible medical wireless capsule. An example of this 


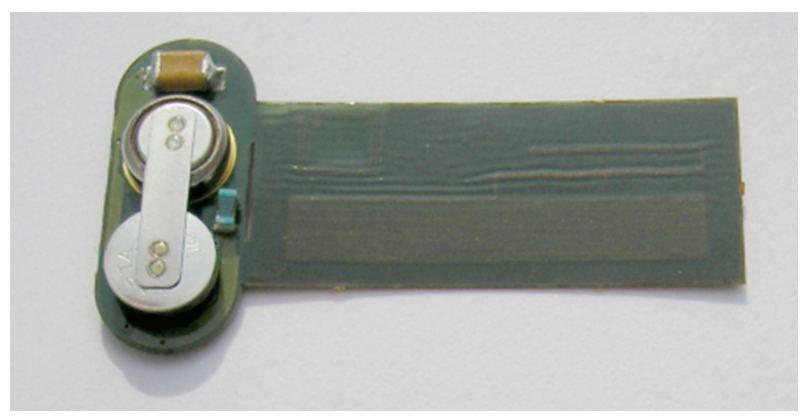

(a)

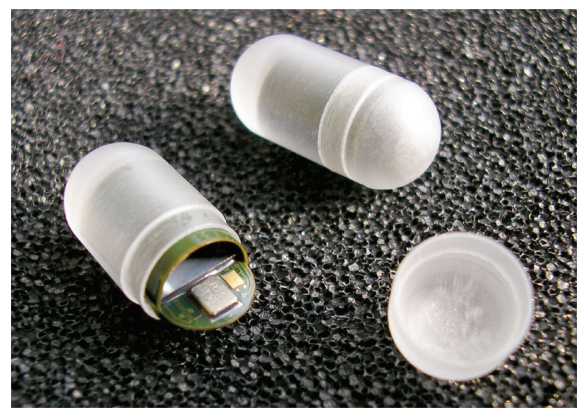

(b)

FIgURE 11: Structure of the system.

antenna has been achieved showing the interest of such an approach. A good quality link from pill embedding conformal antenna to a receiver shows the feasibility of this kind of antenna.

\section{Acknowledgment}

The authors would like to thank NXP Semiconductors for their financial support and for their efforts in manufacturing and test.

\section{References}

[1] Q. Wang, K. Wolf, and D. Plettermeier, "An UWB capsule endoscope antenna design for biomedical communications," in Proceedings of the 3rd International Symposium on Applied Sciences in Biomedical and Communication Technologies (ISABEL '10), pp. 1-6, Rome, Italy, November 2010.

[2] D. Zhao, X. Hou, X. Wang, and C. Peng, "Miniaturization design of the antenna for wireless capsule endoscope," in Proceedings of the 4th International Conference on Bioinformatics and Biomedical Engineering (ICBBE'10), Chengdu, china, June 2010.

[3] P. M. Izdebski, H. Rajagopalan, and Y. Rahmat-Samii, "Conformal ingestible capsule antenna: a novel chandelier meandered design," IEEE Transactions on Antennas and Propagation, vol. 57, no. 4, pp. 900-909, 2009.

[4] S. H. Lee and Y. J. Yoon, "A dual spiral antenna for ultra-wideband capsule endoscope system," in Proceedings of the IEEE International Workshop on Antenna Technology: Small Antennas and Novel Metamaterials (IWAT '08), pp. 227-230, Chiba, Jaban, March 2008.
[5] S. H. Lee, J. Lee, Y. J. Yoon, S. Park, C. Cheon, and S. Nam, "A wideband spiral antenna for ingestible capsule endoscope systems: experimental results in a human phantom and a pig," IEEE Transactions on Biomedical Engineering, vol. 58, no. 6, pp. 1734-1741, 2011.

[6] J. George, M. Deepukumar, C. K. Aanandan, P. Mohanan, and K. G. Nair, "New compact microstrip antenna," Electronics Letters, vol. 32, no. 6, pp. 508-509, 1996.

[7] L. Desclos, Y. Mahe, S. Reed, G. Poilasne, and S. Toutain, "Patch antenna size reduction by combining inductive loading and short-points technique," Microwave and Optical Technology Letters, vol. 30, no. 6, pp. 385-386, 2001.

[8] P. Bhartia, I. Bahl, R. Garg, and A. Ittipiboon, Microstrip Antenna Design Handbook, Artech House Antennas and Propagation Library, Norwood, Mass, USA, 2001.

[9] J. H. Lu and K. L. Wong, "Slot-loaded, meandered rectangular microstrip antenna with compact dual-frequency operation," Electronics Letters, vol. 34, no. 11, pp. 1048-1050, 1998.

[10] Y. Mahe, L. Desclos, R. Moreau, and S. Toutain, "Miniaturized patch antenna for dual-frequency operation," in Proceedings of the 31st European Microwave Conference (EuMC '01), London, UK, September 2001.

[11] C. S. Lee and K. H. Tseng, "Size reduction of microstrip antennas," Electronics Letters, vol. 37, no. 21, pp. 1274-1275, 2001.

[12] A. H. Chaloupa, N. Klien, M. Peiniger, H. Piel, A. Pischke, and G. Splitt, "Miniaturized hightemperature supraconductor patch antenna," IEEE Transaction on Microwaves Theory and Technique, vol. 39, no. 9, pp. 1513-1521, 1991.

[13] M. Kirschning and R. H. Jansen, "Accurate model for effective dielectric constant of microstrip with validity up to millimeter wave frequencies," Electronics Letters, vol. 18, no. 6, pp. 272$273,1982$. 

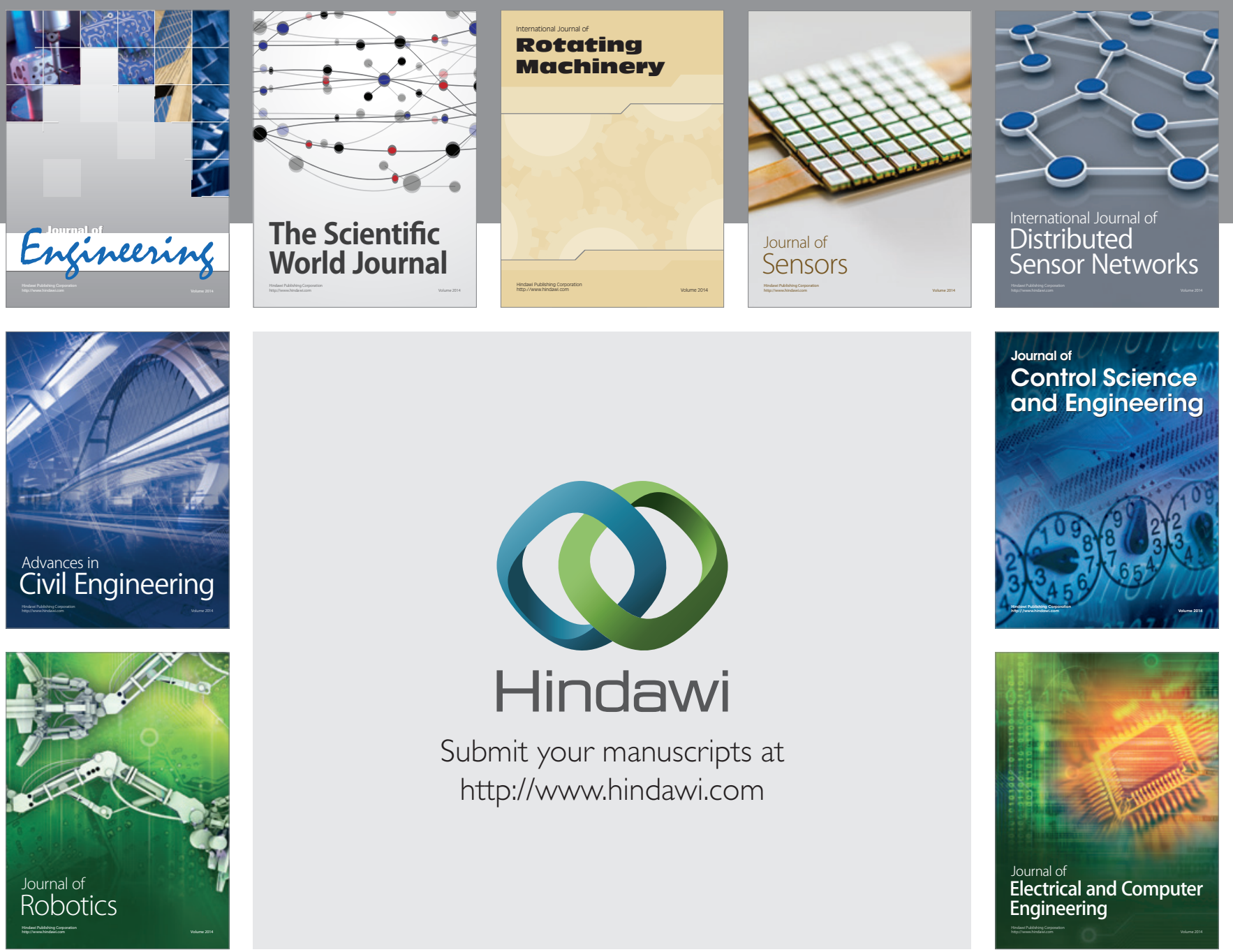

Submit your manuscripts at

http://www.hindawi.com
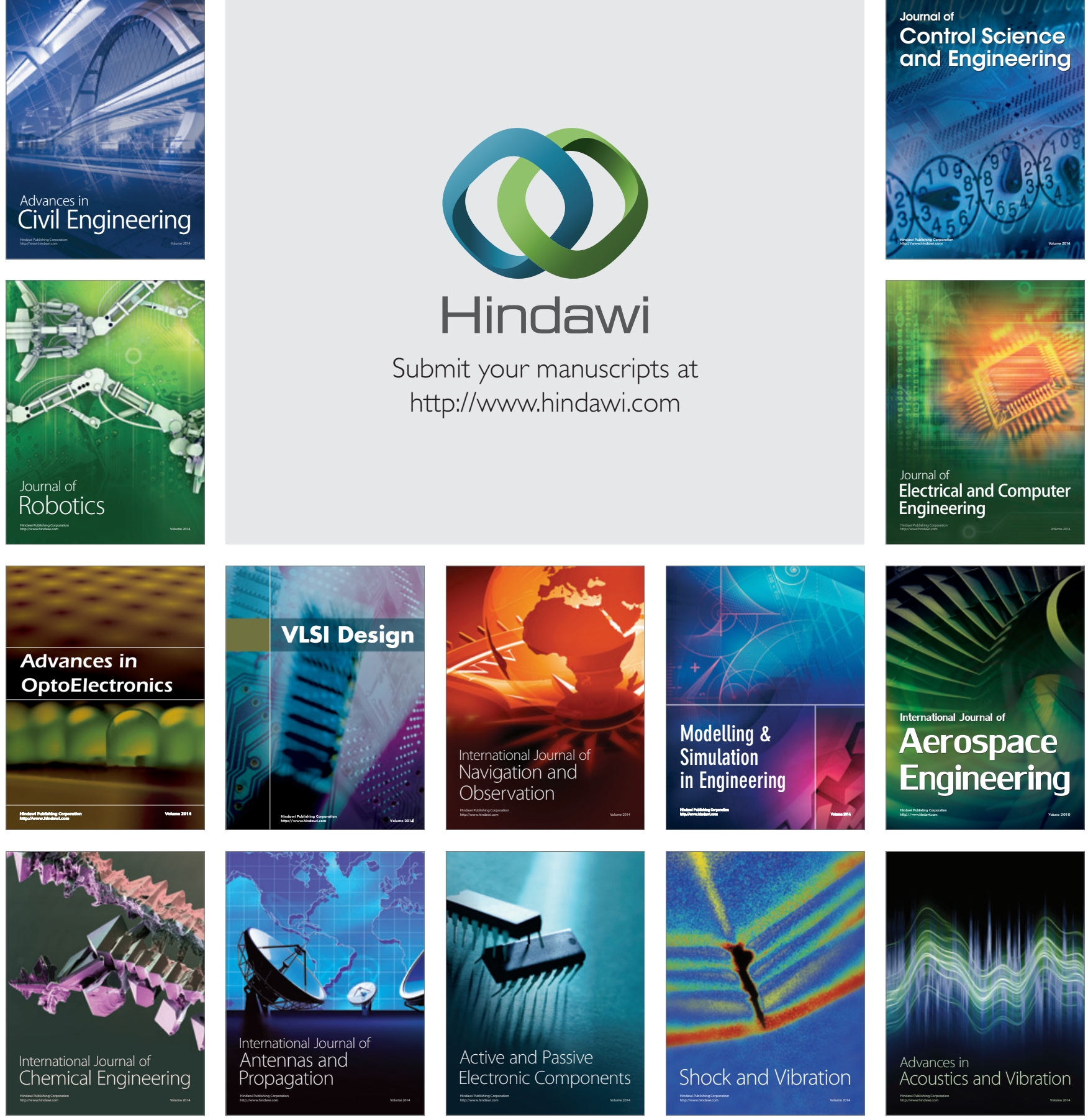